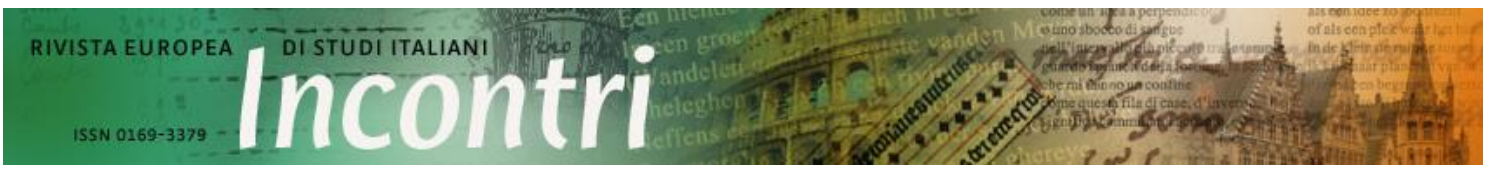

Anno 36, 2021 / Fascicolo 1 / p. 5-6 - www.rivista-incontri.nl - http://doi.org/10.18352/inc11013 (c) The author(s) - Content is licensed under a Creative Commons Attribution 3.0 Unported License Publisher: Werkgroep Italië Studies, supported by openjournals.nl

\title{
Editoriale
}

\section{L’anno di passaggio (alla nuova piattaforma)}

\author{
Natalie Dupré \& Inge Lanslots
}

\author{
Ma la televisione ha detto che il nuovo anno \\ Porterà una trasformazione \\ E tutti quanti stiamo già aspettando \\ L'anno che sta arrivando tra un anno passerà \\ lo mi sto preparando, è questa la novità \\ (Lucio Dalla, L'anno che verrà)
}

La redazione di Incontri è lieta di presentare il primo numero di quest'annata che esordisce sulla nuova piattaforma di Open Journals (openjournals.nl). Ringraziamo Sasha Goldstein-Sabbah e Hans Spijker per l'imprescindibile supporto tecnico e morale durante la fase di transizione.

Per la realizzazione di questo numero teniamo a ringraziare Daniele Comberiati e Alessandra Giro, i due redattori ospiti che si sono presi l'impegno di curare la sezione tematica proponendo uno strumento metodologico innovativo per lo studio della letteratura occidentale. Nell'introduzione Comberiati e Giro si interrogano su due categorie interpretative, lo spaesamento e lo straniamento, definendole come storicamente e contestualmente adattabili. Tale malleabilità storica, esplorata in un arco di tempo compreso tra il XVII e il XIX secolo, abbraccia sia le tecniche narrative sia i nuclei tematici.

Nel primo contributo intitolato 'Una ripetizione straniante. L'idea fissa in un racconto di I.U. Tarchetti' Claudia Murru esplora ulteriormente gli snodi teorici centrali articolati nella fitta introduzione di Comberiati e Giro, snodi che vengono analizzati attraverso il confronto tra i racconti di Tolstoj, di Tarchetti e di Poe.

'Naufragare per giardini. Straniamento e spaesamento nell'Isola Felsenburg di Johann Gottfried Schnabel' di Emanuela Ferragamo si incentra sul rapporto tra protagonista e natura. L'analisi del racconto utopico dimostra quanto Schnabel tenti di addomesticare l'alterità, laddove la narrativa di Tarchetti tende a evocare un senso di straniamento dal quotidiano.

Ellen Patat, a sua volta, prende in esame il topos dello spaesamentoallontanamento nella letteratura odeporica del XIX secolo. Mentre nella sua analisi di genere esplora come le autrici approfondiscano la nozione del nord, Patat include anche riflessioni sulla nozione di letteratura femminile.

"“Coi libri o col mondo?". Effetti di straniamento e spaesamento mancato nel Viaggio e maravigliose di Francesco Contarini' di Bianca Del Buono è dedicato al rapporto tra straniamento ed esperienze di viaggio, rapporto che comporta sia un'ibridazione fra generi diversi sia una pratica di scrittura inter- o metatestuale. 
In ' $L$ 'amour fou tra straniamento e spaesamento in due scrittrici siciliane: Rosina Muzio Salvo e Cettina Natoli' Daniela Bombara esamina il topos dell'amore-passione nella scrittura femminile del Risorgimento e, in particolare, nell'ottica di due scrittrici siciliane poco note.

L'ultimo saggio della sezione tematica, 'Straniamenti e spaesamenti di Luigi Gualdo' di Filippo Fonio, si sviluppa lungo due assi teorici: la relazione fra proiezione ideale e realtà e le problematiche legate alla percezione

Oltre alla sezione tematica questo numero di Incontri presenta un omaggio ad Annaserena Ferruzzi. Il traduttore-scrittore Frans Denissen nel suo 'In memoriam' ci ricorda quanto sia stato importante l'impegno di Ferruzzi nella diffusione della letteratura italiana in olandese.

Il numero si chiude con le recensioni di Stefania Lucamante e di Rachelle Gloudemans, rispettivamente di Finzioni biografiche. Teoria e storia di un genere ibrido di Riccardo Castellana (2019) e di Transcultural Italies curato da Charles Burdett, Loredana Polezzi e Barbara Spadaro (2020).

Auguriamo a tutti un piacevole momento di lettura in quest'anno in cui le misure - speriamo - continueranno ad allentarsi progressivamente.

Natalie Dupré

KU Leuven

Campus Brussel

Warmoesberg 26

1000 Brussel (Belgio)

natalie.dupre@kuleuven.be
Inge Lanslots

KU Leuven

Campus Antwerpen

Sint-Andriesstraat 2

2000 Antwerpen (Belgio)

inge.lanslots@kuleuven.be 